\title{
The Global Math Project: Uplifting Mathematics for All
}

\section{James Tanton and Brianna Donaldson}

\author{
Communicated by Ben Braun
}

ABSTRACT. The Global Math Project aims to connect millions of students around the world through a shared experience of mathematics. For the past year a leadership team of seven math professionals, together with a worldwide network of ambassadors and partners, has been laying the groundwork for the inaugural Global Math Week, which will launch on 10.10.2017.

Here is a bold and audacious idea: let's generate a fundamental paradigm shift as to how the world perceives and enjoys mathematics. Can each and every person on this planet come to see mathematics as human, relevant, meaningful, creative, uplifting, and joyful? Can the play and wonder of mathematics transcend borders and truly unite communities?

\section{Welcome to the Global Math Project}

The vision just outlined is not without precedent. In 2013 the organization code . org set out to bring computer coding to students all across the globe. For one special week that year, millions of students took part in their Hour of Code program, each enjoying some activity-be it a relevant pencil and paper exercise or an advanced interactive online computing experience-that teaches an aspect of coding. The program has been going strong ever since then and has now reached over a quarter of a billion students.

James Tanton is the Mathematical Association of America's mathematician-at-large. His e-mail address is tanton . math@gmai 1 . com.

Brianna Donaldson is director of special projects at American Institute of Mathematics. Her e-mail address is brianna@ai math . org.

For permission to reprint this article, please contact:

reprint-permission@ams . org.

DOI: http://dx.doi.org/10.1090/noti1547

\section{Why Not Do the Same for Math?}

There is one substantial challenge, and it's a perception issue. For many cultures, the idea of signing up for an extracurricular hour of math, as opposed to programming, is decidedly unappealing.

On the other hand, students all across the globe are already engaged in mathematics four or five times a week each and every school week. We can reach millions of students by reaching out to just tens of thousands of teachers, homeschool leaders, math club and math circle leaders, and the like. So why not declare a special week of each year as Global Math Week and
Let's foster a global conversation about joyous mathematics! ask educators to share with their students one engaging and astonishingly thrilling piece of mathematics sometime that week? Let's have students and teachers share their reactions, thoughts, and ideas with comments, videos, or photos posted on social media. Let's foster a global conversation about joyous mathematics!

\section{Enter the Global Math Project}

For the past year a leadership team of seven math professionals has been laying the groundwork to turn the week starting 10.10.2017 into the inaugural Global Math Week (see box on page 715). We believe that the following ingredients are key to the success of Global Math Week 2017:

- a compelling topic,

- appealing and accessible resources for engagement,

- a global network of ambassadors committed to spreading the word, and

- partner organizations that support project activities through financial and in-kind donations. 
Below we describe how these ingredients will combine to create an enthralling global experience of mathematics.

\section{A Compelling Topic: Exploding Dots}

Fundamental to the success of Global Math Week is presenting a mathematical experience that is directly relevant to the standard school content to show that even elementary school topics serve as portals to universes of wonder and delight. We want to show how math, as one youngster recently put it, is "continuous" and keeps inviting further exploration. The mathematics topic should transcend language and the details of any particular curriculum while at the same time fostering an appreciation of fundamental math concepts. It should fit naturally into any classroom.

The team has identified several mathematical storylines that will serve well and have settled on presenting one in particular, Exploding Dots, as the topic for the inaugural experience:

Here is a story that isn't true.

When I was a child I invented a machine (not true) that was nothing more than a series of boxes that could hold dots. And these dots would, upon certain actions, explode. And with this machine, in this nontrue story, I realized I could explain true things! In one fell swoop I explained all the mathematics of arithmetic I learned in grade school (true), all of the polynomial algebra I was to learn in high school (true), elements of calculus and number theory (true)-and also begin to explore unanswered research questions intriguing mathematicians still to this day (also true)!

Let me share this story with you. See how simple and elegant ideas connect to profound ideas in mathematics as a whole. See how these ideas will completely revolutionize your thinking of school arithmetic and algebra and beyond!

So begins the story of Exploding Dots, as developed in 2005 by Global Math Project founder James Tanton. Tanton drew inspiration from a "chip firing" model developed by German educationalist Arthur Engel in the 1970s to explain elementary probability to school students, as well as from mathematician James Propp's efforts to popularize Engel's work. Simple example configurations of Engel's model match the workings of an abacus and illustrate the mechanics of place value, but in all types of bases. Mathematicians have explored the ramifications of arithmetic in these fractional, negative, and irrational bases, and open questions remain [1]. Tanton turned these abacus models back to reconnect them with the $\mathrm{K}-12$ curriculum, in particular moving beyond just place value and arithmetic of primary and middle school to the more advanced

\footnotetext{
${ }^{1}$ See video at $\overline{h t t p s: / / w w w . y o u t u b e . c o m / w a t c h ? v=}$
} hB6bfw622fo mathematics of high school algebra and precalculus, and elements of undergraduate work such as discovering the generating function of the Fibonacci numbers or exploring the 10 -adic representation of negative one.

Exploding Dots is typically introduced by exploring a $1 \leftarrow 2$ machine, which consists of a row of boxes, extending as far to the left as one pleases. To operate this machine, one places a number of dots in the rightmost box, which the machine then redistributes according to the following rule: Two dots in any one box are erased (they "explode"kapow!) to be replaced with one dot located one box to their left. For instance, placing six dots into a $\mathbf{1} \leftarrow \mathbf{2}$ machine yields four explosions, with a final distribution of dots that can be read as "1 1 0." See Figure 1.

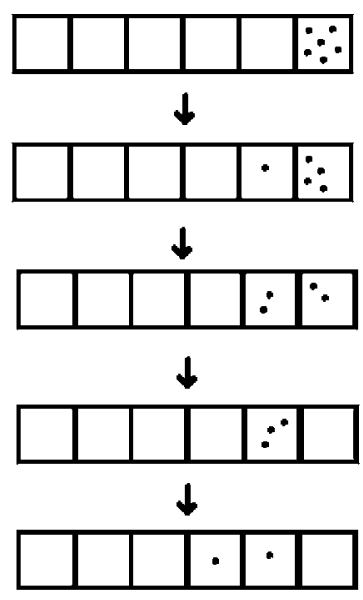

Figure 1: Placing six dots into a $1 \leftarrow 2$ machine yields four explosions, with a final distribution of dots that can be read as " 110. ."

Of course, since one dot in a cell is deemed equivalent to two dots in the preceding cell, each cell is "worth" double the cell to its right. If we deem the rightmost cell as the units, then each cell of the machine corresponds to the powers of two. Thirteen, for instance, equals $8+4+1$, and the base- 2 representation of 13 is 1101 . See Figure 2 .

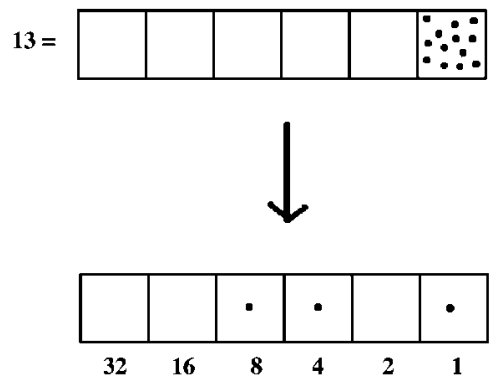

Figure 2: The $1 \leftarrow 2$ machine converts all numbers to their binary representations. Thirteen, for instance, equals $8+4+1$, and the base- 2 representation of 13 is 1101.

The $\mathbf{1} \leftarrow \mathbf{2}$ machine converts all numbers to their binary representations. In the same way, a $\mathbf{1} \leftarrow 3$ machine (three dots explode to make one dot to the left) gives the base-3 


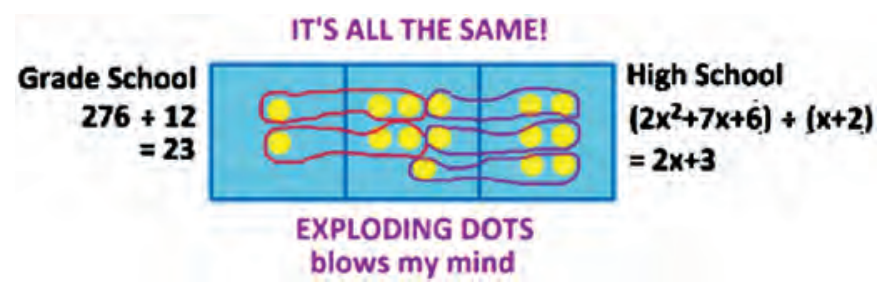

Figure 3: Exploding Dots can explain the mathematics of grade school arithmetic and high school polynomial algebra.

representation of a number and a $\mathbf{1} \leftarrow \mathbf{1 0}$ machine the base10 representation. Taking it a step further, an abstract $\mathbf{1} \leftarrow \mathbf{x}$ machine gives the polynomials of high school algebra.

Exploding Dots is an extraordinary gateway to introduce the habits of mind for doing substantive mathematics-exploring, discovering, and explaining pattern and structure, and then utilizing one's newfound understanding for further exploration and discovery; developing perseverance in pursu-

Exploding Dots is both poetic and utilitarian. ing questions and not settling for half-formed explanations; working with both the abstract and the concrete translation of the abstract; and engaging in a joyful intellectual pursuit. Exploding Dots is both poetic and utilitarian. It is a genuine blend of beautiful mathematics worthy of exploration for its own sake, just as mathematics is often described by mathematicians, and the practical learning of concrete skills for implementation and application. Mathematics is both things, and Exploding Dots is a prime means to demonstrate both sides of this beautiful coin.

Tanton has delivered workshops and lectures on this topic to audiences all across the globe, and students, teachers, and parents alike consistently describe it as "mind blowing." In fact, the inspiration for Global Math Week came from project co-founder Jill Diniz's experience with introducing Exploding Dots to her teenaged son:

... the next day he sent me a text during the school day which said, "Mom, I just showed Exploding Dots to Sammy and Hunter, and we were like, whoaaa, this blows my mind." Then later that evening he came to my room and wanted to talk about just how mind blowing Exploding Dots was, how it related even to what he was doing in AP calculus.

She adds:

Soon thereafter, I sent a note to James and wanted to talk. I had recently become aware of the Hour of Code project and was inspired to apply this idea to Exploding Dots. We just had to get every kid in the country-in the world, even-doing Exploding Dots and doing it roughly at the same time, creating a shared, mind-blowing experience of mathematics that could serve to improve the everyday connection with math for millions of students.

A version of all the Exploding Dots content is currently available on the Web [1].

\section{Appealing and Accessible Resources for Engagement}

So, now we have our inaugural topic, but what will actually happen the week of 10.10.17?

Beginning in April, we opened registration for teachers, math club and math circle leaders, and other educators and math-outreach specialists who have committed to leading a one-hour experience of Exploding Dots during Global Math Week and to sharing that experience with the world in some way, most typically through social media. Also in April we started revealing our new Exploding Dots platform, so that educators can begin playing with the topic themselves and consult with our ever-growing list of ambassadors if guidance is needed.

During Global Math Week itself, our website will serve as a portal to multiple modalities of experiencing Exploding Dots, ranging from low-technology to technologyintensive alternatives. The low-technology experience will consist of downloadable pdfs with lesson plans for leading a discussion about Exploding Dots without any specialized classroom technology (Tanton typically uses only a whiteboard). The more technology-intensive options will use an appealing user interface presented as a collection of "islands" that each represent a particular Exploding Dots topic. Each island will contain three elements to experience: a short explanatory video, a teacher discussion guide, and an interactive Web applet. For those who wish to delve deeper, additional materials will be freely available on the Global Math Project website, created both by the Global Math Project team as well as by interested members of the mathematical community. These materials will support further exploration of place value, arithmetic algorithms, negative numbers, alternative bases, polynomials, formal infinite series, and more.

The entire storyline of Exploding Dots, of course, cannot be completed in just a single class period, but our hope is that teachers and students will be inspired to explore beyond the first few lessons and perhaps to incorporate aspects of the Exploding Dots lessons into their curriculum at other times during the year.

\section{A Global Network of Ambassadors}

A global network of volunteer ambassadors ${ }^{2}$ will play a vital role in spreading the word about the Global Math Project and ensuring access for teachers around the globe by:

- posting about the Global Math Project on social media,

- assisting in forming partnerships with key teacher organizations around the world,

- facilitating translation of Global Math Week content

2https://www. theg1obalmathproject.org/ambassadors 
- hosting webinars to help train teachers on the Exploding Dots materials, and

- organizing public events in their home locations.

By the start of 2017, over 140 people representing more than forty countries had already signed on as Global Math Project Ambassadors. That number has since grown.

We have been in conversation with a number of international mathematics educator societies who want to help spread the word, and we have received photographs and videos from teachers and students all over the world who are already playing with Exploding Dots and eager to share.

\section{Partner Organizations}

Global Math Project Partner Organizations ${ }^{3}$ make significant contributions to the underlying structure of the project, for example, through developing online interfaces and tools, translating materials, promoting Global Math Week to teachers and the public, hosting public events, and providing financial support. The project is hosted by the American Institute of Mathematics (AIM), one of the National Science Foundation-supported mathematical science research institutes, whose mission is to advance mathematics through collaborative problem solving.

Other founding partner organizations include:

- Great Minds, a nonprofit organization that offers content-rich curriculum and professional development

- Math Plus Academy, an after-school mathematics enrichment program focused on problem solving

- The National Museum of Mathematics, the nation's only museum focused on math and its many connections to the world around us

- Scolab, a Canadian educational technology company

Although we have yet to secure major funding, the project has generated much interest and significant in-kind services from partner organizations. We are honored to have these organizations publicly sharing their support for our efforts and welcome additional partner organizations.

\section{How Can Mathematicians and Math Departments Get Involved?}

There are multiple ways that individual mathematicians can become involved in the Global Math Project:

- introduce Exploding Dots to your own students and to K-12 students and teachers with whom you work, through outreach activities

- share information about the Global Math Project with K-12 colleagues and offer to help out when they introduce Exploding Dots to their students

- lead a public Global Math Week event for your community
- become an official Global Math Project Ambassador

- make a financial contribution to the project

Math departments might consider organizing training for local K-12 teachers who want to participate, or hosting a public event during Global Math Week.

\section{What about Next Year?}

Each year we plan to unveil a new Global Math Week topic with a wonderful, "mind-blowing" storyline deeply relevant to the school curriculum, while also keeping previous Global Math Week topics accessible in perpetuity. So Global Math Week 2018 will invite students and teachers and math professionals from all across the globe to try out a new second exciting topic or to experience Exploding Dots. And for 2019 a third topic will be added. And so on.

There is an infinite universe of wondrous mathematics to explore. Let's help communities of folks across the globe experience and share this joy with one another.

\section{Founding Members of the Global Math Project:}

- James Tanton, Mathematician-at-Large, Mathematical Association of America (MAA)

- Jill Diniz, Director of Mathematics Curriculum, Great Minds

- Cindy Lawrence, Executive Director, National Museum of Mathematics (MoMath)

- Brianna Donaldson, Director of Special Projects, American Institute of Mathematics (AIM)

- Derarca Lynch, mathematics instructor, New York University-Abu Dhabi

- Raj Shah, founder and CEO, Math Plus Academy

- Travis Sperry, Director of Information Technology, Math Plus Academy

\section{Reference}

[1] J. TANTON, Exploding Dots, Experience 9, gdaymath.com /courses/exploding-dots/.

\section{ABOUT THE AUTHORS}

James Tanton believes that mathematics really is accessible to all and is committed to sharing the delight and the beauty of the subject through his books, online courses, and lectures. Currently the mathematician-at-large for the Mathematical Association of America, he conducts teacher and student

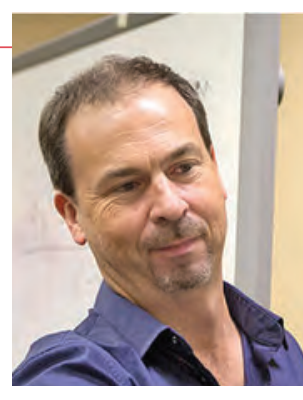
workshops around the world.

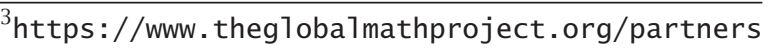




\section{COMMUNICATION}

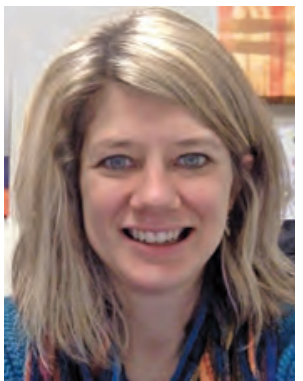

Brianna Donaldson is director of special projects at the American Institute of Mathematics, where she works to broaden participation in mathematics and encourage collaboration through programs such as the Math Teachers' Circle Network, Research Experiences for Undergraduate Faculty, and the Global Math Project.

\section{Brianna Donaldson}

\section{Photo Credit}

Photo of James Tanton is courtesy of the Mathematical Association of America.

Photo of Brianna Donaldson is courtesy of Brianna Donaldson. Figures 1-3 are courtesy of James Tanton.
American Mathematical Society

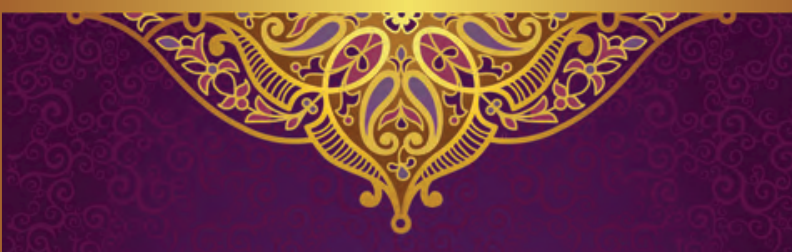

FEATURED TITLES FROM \begin{tabular}{ll}
$\square \square$ & HINDUSTAN \\
\hline$\square$ & BOOK AGENCY
\end{tabular}

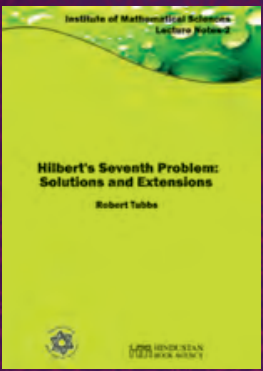

\section{HILBERT'S SEVENTH} PROBLEM

Solutions and Extensions

Robert Tubbs, University of Colorado, Boulder, $\mathrm{CO}$

This exposition is primarily a survey of the elementary yet subtle innovations of several mathematicians between 1929 and 1934 that led to partial and then complete solutions to Hilbert's Seventh Problem (from the International Congress of Mathematicians in Paris, 1900).

Hindustan Book Agency; 2016; 94 pages; Softcover; ISBN: 978-9380250-82-3; List US\$28;AMS members US\$22.40; Order code HIN/72

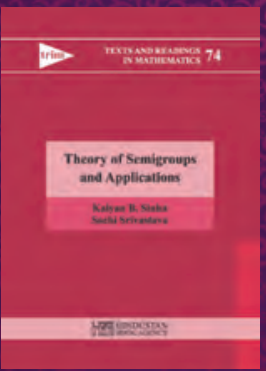

\section{THEORY OF}

SEMIGROUPS AND APPLICATIONS

\section{Movie Review: "Gifted"}

Gifted (2017) is a sweet movie about Mary, a mathematical prodigy, raised by her uncle. Her mother was apparently close to solving the Navier-Stokes million-dollar millennium problem before she committed suicide. Mary amazes her first-grade teacher by computing $57 \times 135$ in her head. Her ambitious grandmother takes her to MIT, where she is challenged to compute

$$
\int_{-\infty}^{\infty} e^{-x^{2} / 2 \sigma^{2}} d x
$$

Later she attends a lecture on Ramanujan and partition functions by a professor played by mathematician Jordan Ellenberg. ${ }^{1}$ I won't spoil the surprise ending.

\section{-Frank Morgan}

${ }^{1}$ See the the interview of Ellenberg in this issue of $\mathrm{No}^{-}$ tices (page 779).
Kalyan B. Sinha, Jawaharlal Nehru Centre for Advanced Scientific

Research, Bangladore, India, and Sachi Srivastava, University of Delhi South Campus, New Delhi, India Combining the spirit of a textbook with that of a monograph on the topic of semigroups and their applications, this book will appeal to readers interested in operator theory, partial differential equations, harmonic analysis, probability and statistics, and classical and quantum mechanics.

Hindustan Book Agency; 2017; 180 pages; Hardcover; ISBN: 978-9386279-63-7; List US\$38;AMS members US $\$ 30.40$; Order code HIN/73

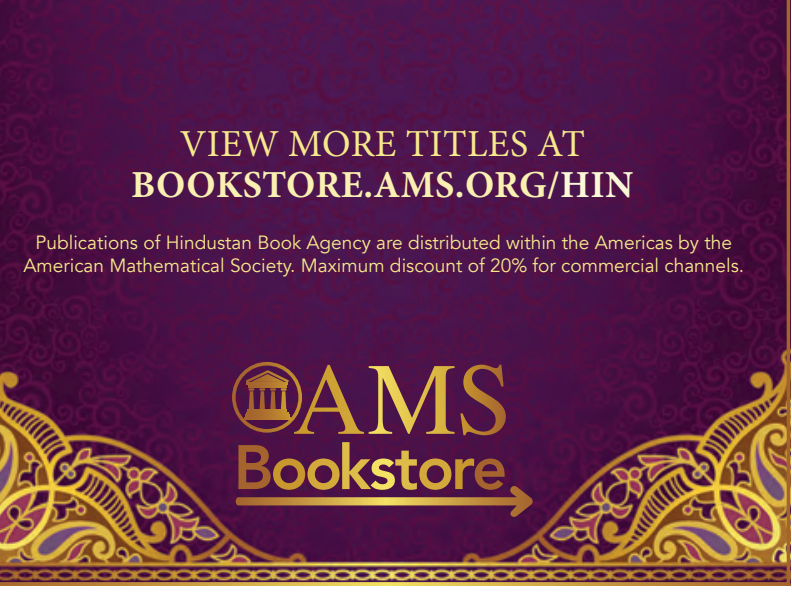

